\title{
Study on the Adaptive Generalized Synchronization of Hetero Structure Chaotic Systems
}

\author{
Chamika Dias ${ }^{1}$, Shu-Yong LIU ${ }^{1, a}$, Jiang JIAN² ${ }^{2}$, Pan SU1 ${ }^{1}$, Xiu-Lei WEI ${ }^{1}$, \\ Qing-Chao YANG ${ }^{1}$, Yuan FANG ${ }^{1}$ \\ ${ }^{1}$ College of Power Engineering, Naval University of Engineering, Wuhan, China \\ IInstitute of Acoustic, Chinese Academy of Sciences, Beijing, China
}

Keywords: Vibration system, Nonlinear dynamics, Parameter monitor, Attractor.

\begin{abstract}
A mathematical model of adaptive generalized chaotic synchronization(AGCS) was established to investigate the synchronization between a multi-degree freedom non-autonomous(nonlinear vibration) driving system and an autonomous chaotic (Lorenz) response system. Within the hetero structure synchronization model, The AGCS nonlinear adaptive controller is designed. Both the AGCS error curves and parameters identification error curves are proved to converge to zero by Lyapunov stability theory. Numerical investigations were carried out to obtain the synchronization characteristic pattern. Results show that these two systems can be synchronized by using AGCS technique, with both the synchronization error and the parameter identification error quickly converging to zero. The chaotic attractor is observed in phase plane, and the synchronization diagonal line is applied to show the existence of the synchronized behavior. The model established in this study can be used for condition monitoring and fault diagnosis of chaotic vibration systems in many engineering fields.
\end{abstract}

\section{Introduction}

Since 1990, when the first experimental study of chaos synchronization (CS) was conducted successfully by Pecora and Carroll1, CS has been widely applied in many fields such as security communication, ultra-wideband wireless transmission and chaos anti-controlling.

In order to improve the performance of synchronization between two chaotic systems, many studies have been carried out focusing on improving the efficiency of controller2-14.

For two chaotic systems with same structure, Tan9 designed a new nonlinear feedback controller based on fractional Laplace transform theory. This controller can be used for generalized synchronization between fractional-order chaotic systems. A criterion for synchronizing two chaotic systems was also given. Niu10 investigated the self-synchronization of variable coefficients chaotic system, in which a controlling method with strictly positive real transfer function matrix was used. Zeng11 studied the global asymptotic synchronization of chaotic systems with the same structures. The controller was designed based on radial basis function neural network.

A great number of studies were also conducted focusing on the synchronization of different structures chaotic systems. For example, Liu 12 investigated the active disturbance rejection controller for anti-synchronization between chaotic systems with different structures. Chen13 studied the adaptive generalized synchronization (AGS) between a Chen system and a multi-scroll chaotic system. Sun 14 studied the complexity of generalized synchronization (GS) between two unidirectional coupled Lorenz systems under parametric excitation.

Although many researches have been conducted on the subject of chaotic synchronization, most of them were focusing on autonomous chaotic systems. No investigation of the adaptive generalized synchronization between multi-degree-freedom non-autonomous and autonomous systems has been carried out, though the non-autonomous systems are widely used in practice15-20. It is very important to extend the study and application of AGCS to the non-autonomous systems.

In Sec.2, a mathematic model was established to investigate the chaos synchronization between a multi-degree freedom non-autonomous (nonlinear vibration) system and an autonomous chaotic (Lorenz) system. In Sec.3, an adaptive controller was designed to synchronize the two systems. The 
convergences of both synchronization error and parameter identification error were also studied using the Lyapunov theory. In Sec.4, the AGCS between these two systems were simulated and the reliability of using this model to detect and monitor parameter variations is investigated. Conclusions were given in Sec.6.

\section{Adaptive Generalized Synchronization Model for Chaotic Systems}

An AGCS model was designed to synchronize a multi-degree freedom non-autonomous (nonlinear vibration) driving system and an autonomous chaotic (Lorenz) response system.

The driving system used in the model has two degree of freedoms, as seen in Fig. 1. It includes a nonlinear stiffness spring between an upper mass $\mathrm{m} 1$ and the middle mass; a linear stiffness spring between a middle mass $\mathrm{m} 2$ and a foundation. This system has been previously studied by Yu Xiang et al.21 In his paper where the characteristics of nonlinear stiffness was carefully investigated.

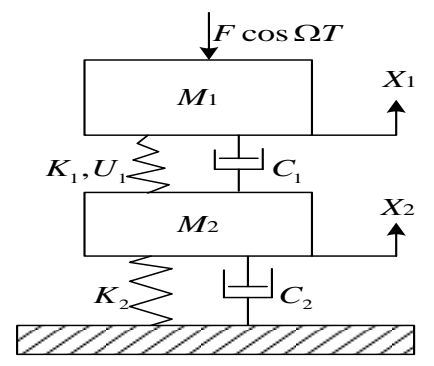

Fig.1. Two-degree-freedom nonlinear vibration system

The dynamic equation of the system can be expressed as:

$$
\begin{aligned}
& M_{1} \ddot{X}_{1}+C_{1}\left(\dot{X}_{1}-\dot{X}_{2}\right)+K_{1}\left(X_{1}-X_{2}\right)+U_{1}\left(X_{1}-X_{2}\right)^{3} \\
& =F \cos (\Omega T)+G_{0} \\
& M_{2} \ddot{X}_{2}-C_{1}\left(\dot{X}_{1}-\dot{X}_{2}\right)-K_{1}\left(X_{1}-X_{2}\right)-U_{1}\left(X_{1}-X_{2}\right)^{3} \\
& +C_{2} \dot{X}_{2}+K_{2} X_{2}=0
\end{aligned}
$$

where, M1,M2 are upper and lower mass blocks respectively; X1,X2 are vibration displacement; $\mathrm{C} 1, \mathrm{C} 2$ are system damping; $\mathrm{K} 1, \mathrm{~K} 2$ are linear stiffness; $\mathrm{U} 1$ is the nonlinear stiffness; $\mathrm{F}$ is excitation force amplitude; $\Omega$ is the excitation frequency; $G_{0}$ is the gravity of upper mass block and

$$
\ddot{X}_{i}=d^{2} X_{i} / d T^{2}, \dot{X}_{i}=d X_{i} / d T \quad(\mathrm{i}=1,2)
$$

The dimensionless equation is derived from Eq.(1) and gets

$$
\begin{aligned}
& \ddot{x}_{1}+\xi_{1}\left(\dot{x}_{1}-\dot{x}_{2}\right)+\left(x_{1}-x_{2}\right)+\left(x_{1}-x_{2}\right)^{3} \\
& =f \cos (\omega t)+G \\
& \ddot{x}_{2}-\mu \xi_{1}\left(\dot{x}_{1}-\dot{x}_{2}\right)-\mu\left(x_{1}-x_{2}\right)-\mu\left(x_{1}-x_{2}\right)^{3} \\
& +\xi_{2} \dot{x}_{2}+\mu K x_{2}=0
\end{aligned}
$$

where $\quad x_{1}=X_{1} / \sqrt{K_{1} / U_{1}} ; x_{2}=X_{2} / \sqrt{K_{1} / U_{1}} ; t=\Omega_{0} T ; \Omega_{0}=\sqrt{K_{1} / M_{1}} ; f=F / K_{1} \sqrt{K_{1} / U_{1}}$; $\omega=\Omega / \Omega_{0} ; G=G_{0} / K_{1} \sqrt{K_{1} / U_{1}} ; \xi_{1}=C_{1} / \sqrt{K_{1} M_{1}} ; \xi_{2}=C_{2} \mu / \sqrt{K_{1} M_{1}} ; K=K_{2} / K_{1} ; \mu=M_{2} / M_{1}$; $\ddot{x}_{i}=d^{2} x_{i} / d t^{2} ; \dot{x}_{i}=d x_{i} / d t \quad(\mathrm{i}=1,2)$. To simplify the analysis, let $\mu=2$ in Eq.(2), and yields 


$$
\begin{aligned}
& \ddot{x}_{1}+\xi_{1}\left(\dot{x}_{1}-\dot{x}_{2}\right)+\left(x_{1}-x_{2}\right)+\left(x_{1}-x_{2}\right)^{3} \\
& =f \cos (\omega t)+G \\
& \ddot{x}_{2}-2 \xi_{1}\left(\dot{x}_{1}-\dot{x}_{2}\right)-2\left(x_{1}-x_{2}\right)-2\left(x_{1}-x_{2}\right)^{3} \\
& +\xi_{2} \dot{x}_{2}+2 K x_{2}=0
\end{aligned}
$$

In order to better analyze the states of variables, Eq. (3) can be re-written as first-order state equations:

$$
\begin{aligned}
\dot{x}_{1} & =x_{3} \\
\dot{x}_{2} & =x_{4} \\
\dot{x}_{3} & =-\xi_{1}\left(x_{3}-x_{4}\right)-\left(x_{1}-x_{2}\right) \\
& -\left(x_{1}-x_{2}\right)^{3}+f \cos (\omega t)+G \\
\dot{x}_{4}= & 2 \xi_{1}\left(x_{3}-x_{4}\right)+2\left(x_{1}-x_{2}\right) \\
& +2\left(x_{1}-x_{2}\right)^{3}-\xi_{2} x_{4}-2 K x_{2}
\end{aligned}
$$

The equations for response system (Lorenz system) with relevant control components are given as

$$
\begin{aligned}
& \dot{y}_{1}=a\left(y_{2}-y_{1}\right)+u_{1} \\
& \dot{y}_{2}=c y_{1}-y_{2}-y_{1} y_{3}+u_{2} \\
& \dot{y}_{3}=y_{1} y_{2}-b y_{3}+u_{3}
\end{aligned}
$$

where, y1, y2, y3 are system outputs; a, b, c are Lorenz system parameters and u1,u2,u3 are control components which will be further discuss in Sec.3.

To synchronize these two systems, a target function which consists of the state variables in Eq.(4) is given as:

$$
\varphi=\left(\varphi_{1}, \varphi_{2}, \varphi_{3}\right)=\left(x_{1}+x_{3}, x_{2}+x_{4}, x_{1}+x_{2}\right)^{T}
$$

Therefore synchronization errors can be expressed as

$$
e_{1}=y_{1}-\varphi_{1}, \quad e_{2}=y_{2}-\varphi_{2}, \quad e_{3}=y_{3}-\varphi_{3}
$$

The total synchronization error of AGCS is given as $e=\left(e_{1}^{2}+e_{2}^{2}+e_{3}^{2}\right)^{1 / 2}$. The adaptive generalized synchronization between system(4) and system (5) can be achieved when $\lim _{t \rightarrow+\infty} e(t)=0$.

\section{Design of Controller}

The control components in Eq.(5) are designed to be nonlinear and adaptive, as follows:

$$
\begin{aligned}
u_{1}= & (a-1) y_{1}-a y_{2}+x_{1}+\left(2-\hat{\xi}_{1}\right) x_{3} \\
& +\hat{\xi}_{1} x_{4}-\left(x_{1}-x_{2}\right)^{3}+\hat{f} \cos (\omega t)+\hat{G} \\
u_{2}= & -c y_{1}+y_{1} y_{3}+2 x_{1}-(1+2 \hat{K}) x_{2} \\
& +2 \hat{\xi}_{1} x_{3}+\left(2-2 \hat{\xi}_{1}-\hat{\xi}_{2}\right) x_{4}+2\left(x_{1}-x_{2}\right)^{3} \\
u_{3}= & (b-1) y_{3}-y_{1} y_{2}+x_{1}+x_{2}+x_{3}+x_{4}
\end{aligned}
$$

where $\hat{\xi}_{1}, \hat{\xi}_{2}, \hat{f}, \hat{G}, \hat{K}$ corresponding to those in the driven system are unknown parameters need to be identified. The identification errors are expressed as 


$$
\begin{aligned}
& \bar{\xi}_{1}=\hat{\xi}_{1}-\xi_{1}, \bar{\xi}_{2}=\hat{\xi}_{2}-\xi_{2}, \\
& \bar{f}=\hat{f}-f, \bar{G}=\hat{G}-G, \bar{K}=\hat{K}-K
\end{aligned}
$$

Coefficients of the controller are actively adjusted by the synchronization errors. The adaptive laws for parameter identification are given as

$$
\begin{aligned}
& \dot{\hat{\xi}}_{1}=v\left(e_{1}-2 e_{2}\right)\left(x_{3}-x_{4}\right) \\
& \dot{\hat{\xi}}_{2}=v e_{2} x_{4} \\
& \dot{\hat{f}}=-v e_{1} \cos (\omega t) \\
& \dot{\hat{G}}=-v e_{1} \\
& \dot{\hat{K}}=2 v x_{2} e_{2}
\end{aligned}
$$

where $\mathrm{v}$ is the control coefficient used to adjust converging velocity.

To prove the adaptive generalized synchronization can be achieved between these two systems, the Lyapunov function was used to investigate the convergence of errors. Take the Lyapunov function as

$$
V=\frac{1}{2}\left(e_{1}^{2}+e_{2}^{2}+e_{3}^{2}\right)+\frac{1}{2 v}\left(\bar{\xi}_{1}^{2}+\bar{\xi}_{2}^{2}+\bar{f}^{2}+\bar{G}^{2}+\bar{K}^{2}\right)
$$

Combining Eq.(9) with Eq.(11), and thus,

$$
\begin{aligned}
\dot{V} & =e_{1} \dot{e}_{1}+e_{2} \dot{e}_{2}+e_{3} \dot{e}_{3} \\
& +\frac{1}{v}\left(\bar{\xi} \dot{\xi}_{1}+\bar{\xi}_{2} \dot{\hat{\xi}}_{2}+\dot{\hat{f f}}+\bar{G} \dot{\hat{G}}+\bar{K} \dot{\hat{K}}\right)
\end{aligned}
$$

By differentiating Eq.(7) and substituting into Eq. (12), we get:

$$
\begin{aligned}
\dot{V} & =e_{1}\left(\dot{y}_{1}-\dot{\varphi}_{1}\right)+e_{2}\left(\dot{y}_{2}-\dot{\varphi}_{2}\right)+e_{3}\left(\dot{y}_{3}-\dot{\varphi}_{3}\right) \\
& +\frac{1}{v}\left(\bar{\xi}_{1} \dot{\hat{\xi}}_{1}+\bar{\xi}_{2} \dot{\hat{\xi}}_{2}+\dot{\hat{f f}}+\bar{G} \dot{\hat{G}}+\bar{K} \dot{\hat{K}}\right)
\end{aligned}
$$

Substitute Eqs.(4),(5),(6),(9) and Eq.(10) into Eq.(13), and the Eq.(14) is deduced,

$$
\begin{aligned}
\dot{V} & =e_{1}\left[x_{1}-y_{1}+x_{3}+\left(\xi_{1}-\hat{\xi}_{1}\right)\left(x_{3}-x_{4}\right)\right. \\
& +(\hat{f}-f) \cos (\omega t)+(\hat{G}-G)]+e_{2}\left[x_{2}+x_{4}-y_{2}\right. \\
& \left.+\left(\xi_{2}-\hat{\xi}_{2}\right) x_{4}+2(K-\hat{K}) x_{2}-2\left(\xi_{1}-\hat{\xi}_{1}\right) x_{3}\right] \\
& +e_{3}\left(x_{1}+x_{2}-y_{3}\right)-\left(\xi_{1}-\hat{\xi}_{1}\right)\left(e_{1}-2 e_{2}\right)\left(x_{3}-x_{4}\right) \\
& -\left(\xi_{2}-\hat{\xi}_{2}\right) e_{2} x_{4}-(\hat{f}-f) e_{1} \cos (\omega t) \\
& -e_{1}(\hat{G}-G)-2 e_{2}(K-\hat{K}) x_{2} \\
& =e_{1}\left(x_{1}-y_{1}+x_{3}\right)+e_{2}\left(x_{2}+x_{4}-y_{2}\right) \\
& +e_{3}\left(x_{1}+x_{2}-y_{3}\right) \\
& =-\left(e_{1}^{2}+e_{2}^{2}+e_{3}^{2}\right) \\
& =-e^{2} \leq 0
\end{aligned}
$$

Since $\dot{V} \leq 0$, and 


$$
\int_{o}^{t} e^{2} d t=\int_{o}^{t}(-\dot{V}) d t=V(0)-V(t) \leq V(0)
$$

According to Barbalat lemma16, the limitation of $\mathrm{e}(\mathrm{t})$ is $\lim _{t \rightarrow+\infty} e(t)=0$. Then, the synchronization between systems (4) and (5) can be achieved.

Furthermore, the parameters identification errors need to converge to ensure the parameters given in Eq.(4) can be identified effectively. Considering Eq.(7), we can get

$$
\begin{aligned}
& \dot{e}_{1}=\dot{y}_{1}-\dot{\varphi}_{1} \\
& \dot{e}_{2}=\dot{y}_{2}-\dot{\varphi}_{2} \\
& \dot{e}_{3}=\dot{y}_{3}-\dot{\varphi}_{3}
\end{aligned}
$$

Substitute Eqs.(5),(6) into Eq.(15), and obtain

$$
\begin{aligned}
& \dot{e}_{1}=a\left(y_{2}-y_{1}\right)+u_{1}-\dot{x}_{1}-\dot{x}_{3} \\
& \dot{e}_{2}=c y_{1}-y_{2}-y_{1} y_{3}+u_{2}-\dot{x}_{2}-\dot{x}_{4} \\
& \dot{e}_{3}=y_{1} y_{2}-b y_{3}+u_{3}-\dot{x}_{1}-\dot{x}_{2}
\end{aligned}
$$

Then Eq.(17) is derived by substitute Eqs.(4),(8) into Eq.(16),

$$
\begin{aligned}
\dot{e}_{1}= & -e_{1}+\left(\xi_{1}-\hat{\xi}_{1}\right)\left(x_{3}-x_{4}\right) \\
& +(\hat{f}-f) \cos (\omega t)+(\hat{G}-G) \\
\dot{e}_{2} & =-e_{2}+\left(\xi_{2}-\hat{\xi}_{2}\right) x_{4} \\
& +2(K-\hat{K}) x_{2}-2\left(\xi_{1}-\hat{\xi}_{1}\right) x_{3} \\
\dot{e}_{3} & =x_{1}+x_{2}-y_{3}=-e_{3}
\end{aligned}
$$

when $t \rightarrow \infty$,considering the limitation of error $e(t) \rightarrow 0$,thus the corresponding components $e_{1}(t) \rightarrow 0, e_{2}(t) \rightarrow 0, e_{3}(t) \rightarrow 0$. Sincee $1(\mathrm{t}), \mathrm{e} 2(\mathrm{t}), \mathrm{e} 3(\mathrm{t})$ are smooth functions, the derivative of errors are satisfied $\dot{e}_{1}(t) \rightarrow 0, \dot{e}_{2}(t) \rightarrow 0, \dot{e}_{3}(t) \rightarrow 0$.

Therefore, ast approaches infinite, we can get Eq.(18) from Eq.(17)

$$
\begin{aligned}
& \left(\xi_{1}-\hat{\xi}_{1}\right)\left(x_{3}-x_{4}\right)+(\hat{f}-f) \cos (\omega t)+(\hat{G}-G)=0 \\
& \left(\xi_{2}-\hat{\xi}_{2}\right) x_{4}+2(K-\hat{K}) x_{2}-2\left(\xi_{1}-\hat{\xi}_{1}\right) x_{3}=0
\end{aligned}
$$

Substitute Eq. (9) into Eq. (18), and thus

$$
\begin{gathered}
\bar{f} \cos (\omega t)-\bar{\xi}_{1}\left(x_{3}-x_{4}\right)+\bar{G}=0 \\
2 \bar{\xi}_{1} x_{3}-\bar{\xi}_{2} x_{4}-2 \bar{K} x_{2}=0
\end{gathered}
$$

As the state variables of the system (4), ${ }_{2}, x_{3}, x_{4}$ are not identically equal to zero. To ensure the Eq. (20) can be hold under all the states, following conditions must be satisfied, $\bar{\xi}_{1}=\bar{\xi}_{2}=\bar{K}=0$

$$
\hat{\xi}_{1} \rightarrow \xi_{1}, \hat{\xi}_{2} \rightarrow \xi_{2}, \hat{K} \rightarrow K
$$

Then the Eq. (19) can be expressed as

$$
\bar{f} \cos (\omega t)+\bar{G}=0
$$


Since value $\cos (\omega t)$ is not identically equal to zero, to ensure the Eq.(21) is tenable, following conditions must be satisfied, $\bar{f}=\bar{G}=0$, and thus $, \hat{f} \rightarrow f, \hat{G} \rightarrow G$.

In summary, if the controller (8) and adaptive law (10) are adopted, the adaptive generalized synchronization can be achieved. Both the synchronization errors and the parameter identification errors converge to zero.

\section{Numerical Simulation of AGCS}

When parameters $\xi_{1}=0.02, \xi_{2}=0.2, \mathrm{~K}=100, \mathrm{f}=8.8, \omega=3.7, \mathrm{G}=96$, coefficient $\mathrm{v}=15$, giving the initial values of the two-degree freedom non-autonomous chaotic system (4) as

$$
\left[x_{1}(0), x_{2}(0), x_{3}(0), x_{4}(0)\right]=[1,1,1,1]
$$

a chaotic behavior occurs. Relevant $\mathrm{x} 1, \mathrm{x} 2, \mathrm{x} 3, \mathrm{x} 4$ can be obtained by using Runge-Kutta algorithm and the phase diagrams of $x 1, x 3$ and $x 2$, $x$ 4are shown in Fig.2.

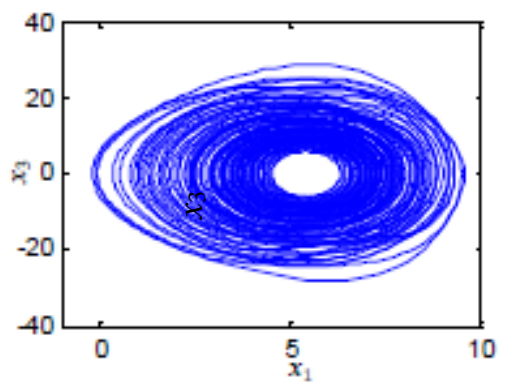

(a)Phase diagram of $x_{1}-x_{3}$

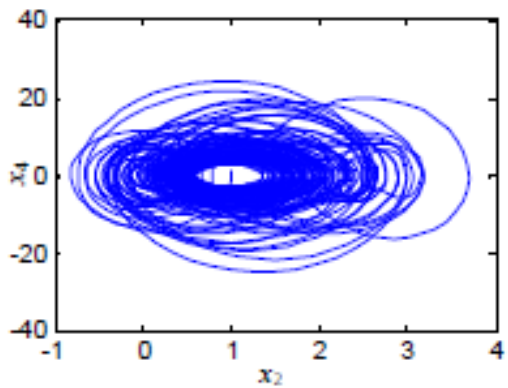

(b)Phase diagram of $x_{2}-x_{4}$

Fig.2.Phase diagram of two-degree freedom on-autonomous chaotic system

The initial values of Lorenz response system, Eq. (5), are given as $\left[y_{1}(0), y_{2}(0), y_{3}(0)\right]=[1,1,1]$ and relevant parameters as $a=10, b=28, c=8 / 3$, The adaptive controller introduced in Sec. 3 is used to synchronize these two systems. To achieve the synchronization, the synchronization errors, e1, e2, e3, and identification errors, $\bar{\xi}_{1}, \bar{\xi}, \bar{f}, \bar{G}, \bar{K}$ should all converge to zero. Results are shown in Fig.3.

From Fig.3, it is clear that all the errors converge to zero after about 20s, when the AGS of these two systems were achieved, as shown in Figs.3 (a) and (b). Under the synchronized status, the AGS synchronization pattern between two systems (in Fig.3.c) stays in a diagonal line. 


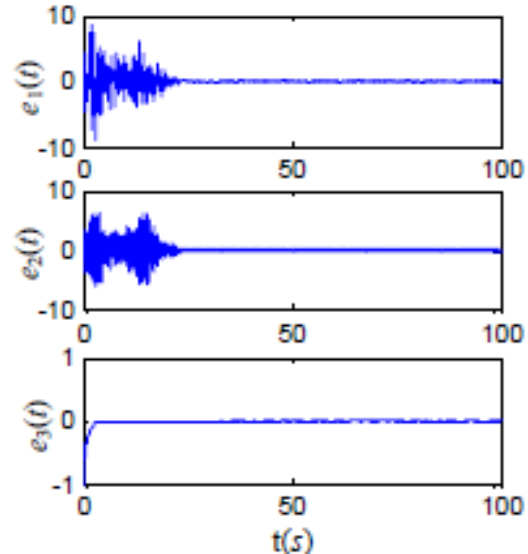

(a) Synchronization errors

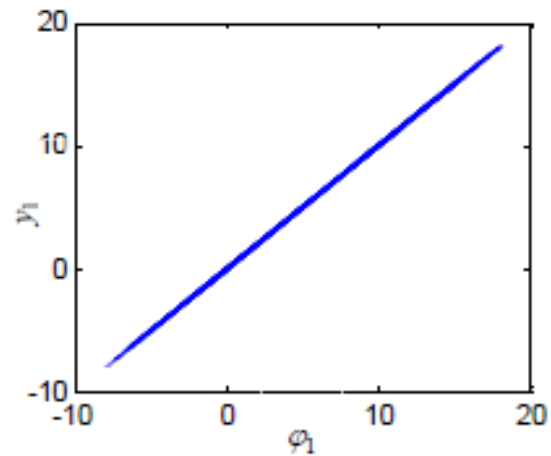

(C) Synchronization pattern between two systems
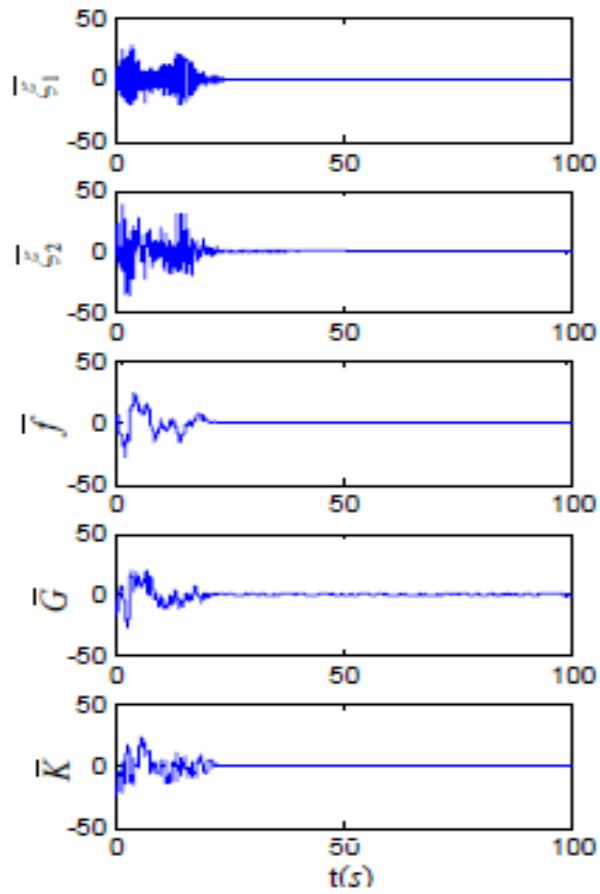

(b) Parameter identification error

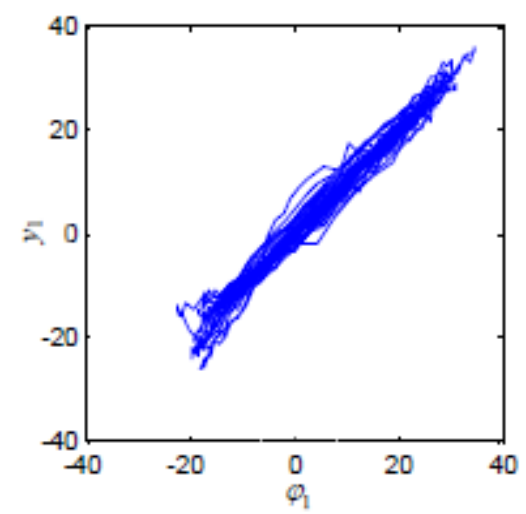

(d) Non-synchronization between two systems

Fig.3.Synchronization process between two systems

\section{Conclusions}

An adaptive generalized chaotic synchronization (AGCS) technique is successfully used to synchronize a two-degree-freedom non-autonomous chaotic (nonlinear vibration) system and an autonomous (Lorenz) system, where the first system is the driving system, and the second one is the response system. The AGCS synchronization frame is given and the nonlinear adaptive controller is designed to achieve the adaptive generalized synchronization. Both the AGCS error curves and parameters identification error curves are proved to converge to zero by Lyapunov stability theory. A numerical simulation was carried out to obtain the synchronization characteristic pattern. It is observed that the AGCS is achieved when errors converge to zero, and the synchronization diagonal line is applied to show the existence of the synchronized behavior. If the diagonal line with slope 1.0 exists, the two systems work in a synchronization state.

\section{Acknowledgement}

Project is supported by Project 425517K143 and the National Natural Science Foundation of China (Grant No. 51579242, 51509253). 


\section{References}

1. Pecora L M, Carroll T L,Physical Review Let ters , 64(8),821-824(1990).

2. Hu J B, Zhao L D,Acta Phys. Sin., 62(24),240504-240504(2013).

3. Zhang X D, Zhu P, Xie X P.A, Acta Phys. Sin. 62(21),21056-21056(2013).

4. Yu H T, Wang J., Acta Phys. Sin. 62(17), 170511-170511(2013).

5. Ma M L, Min F H, Shao S Y, Acta Phys. Sin. 63 (1), 10507 (2014).

6. Hou Z S, Jin S T, IEEE Trans. Neural Networks, 22 (12), 2173-2188(2011).

7. Jens W, Marco A, Leonardo R, Int. J. Control,84(1),138-164 (2011).

8. Bu X H, Yu F S, Zhang H W,Math. Problems Engin., 223(2),199-210 (2012)

9. Tan Wen, Jang Fengling, Wang Yaonan,Computer Engineering \&Science, 35(1), 66-71(2013).

10. Niu H, Zhang G S,Acta Phys. Sin.,62(13),130502-130502(2013).

11. Zeng Zhe-Zhao, Acta Phys. Sin.,62(3),03054-03054 (2009).

12. Liu F C, Jia Y F, Ren L N,Acta Phys. Sin.,62(12),120509-120509 (2013).

13. Chen L, Shi Y D, Wang D S,Chin. Phys. B,10(10), 145-147(2010).

14. Sun Kehui, Wang Yan, Wang Yanli,Journal of Central South University,21(3), 948-955 (2014).

15. Hu A H, Xu Z Y, Guo L X, Acta Phys. Sin. 58(9), 6030-6038(2009).

16. Jiang S M, Tian L X,Acta Phys. Sin. 55(7), 3322-3327 (2006).

17. Larger L, Dudley J M, Nature, 465,41-42 (2010).

18. Yalcin M E, Ozoguz S, Chaos, 17 (3), 033112-033112(2007).

19. N. Vasegh, F. Khellat,Chaos, Solitons \& Fractals, 42(2), 1054-1061(2009).

20. Wang Yue-Gang,Wen Chao-Bin,Acta Phys. Sin., 62(10),100504-100504(2013).

21. Yu Xiang, Zhu Shijian, Shuyong Liu, Journal of Sound and Vibration,306(3-5), 835-848(2007). 